\title{
Heterogeneity of subclinical autistic traits among parents of children with autism spectrum disorder: Identifying the Broader Autism Phenotype with a data-driven method
}

\section{Running title: BAP and LCA in parents}

Emre Bora $^{\mathrm{a}, \mathrm{f}}$, Aydan Aydın ${ }^{\mathrm{b}}$, Tuğba Saraçc, Muhammed Tayyib Kadak ${ }^{\mathrm{d}}$, Sezen Köse ${ }^{\mathrm{e}}$

a-The Melbourne Neuropsychiatry Centre, Department of Psychiatry, The University of

Melbourne and Melbourne Health, VIC, Australia

b-Marmara University, Faculty of Education, Department of Special Education, Istanbul,

Turkey

c-Disk K1z Teknik ve Meslek Lisesi, Istanbul, Turkey

d-Istanbul University Cerrahpasa Medical School, Department of Child and Adolescent

Psychiatry, Istanbul, Turkey

e-Ege University, Department of Child and Adolescent Psychiatry, İzmir, Turkey

f-6328 Sok no:38/2, Yali Mahallesi, Izmir, Turkey

Corresponding author: Emre Bora

ibora@unimelb.edu.au, boremre@gmail.com

Melbourne Neuropsychiatry Centre

Level 3, Alan Gilbert Building

161 Barry St, Carlton

Vic 3053, Australia

Telephone: 61390358628

Fax: 61393480469

Number of pages: 10 (main text)

Number of Tables $=2$

Number of Figures $=2$

\section{Grant information: None}

This is the author manuscript accepted for publication and has undergone full peer review but has not been through the copyediting, typesetting, pagination and proofreading process, which may lead to differences between this version and the Version record. Please cite this article as doi:10.1002/ aur.1661. 


\section{Lay Abstract}

Mild autistic traits are significantly more common among unaffected parents of firstdegree relatives of individuals with autism. However, the severity of autistic traits is not significantly different from the general population in the majority of these parents. In this study, we aimed to identify the subgroup of parents with increased subthreshold autistic traits by a data-driven method called latent class analysis. We analysed Autism Spectrum Quotient (AQ) ratings of 673 parents of children with ASD and 147 parents of typically developing children. Two distinct subgroups, including a "low-scoring" and a "high-scoring" groups, were found. In comparison to control parents, a significantly larger proportion (21.1\% vs $7.5 \%$ ) of parents of ASD were members of the"highscoring" group. Self-reported communication difficulties made a distinctive contribution to the separation of high and low-scoring groups. 


\begin{abstract}
:
Clinical diagnosis of autism spectrum disorder (ASD) can be conceptualized as the extreme end of the distribution of subclinical autistic traits related to genetic susceptibility factors (broad autism phenotype (BAP)) in the general population. Subclinical autistic traits are significantly more common among unaffected first-degree relatives of probands with autism. However, there is a significant heterogeneity of autistic traits in family members of individuals with ASD and severity of autistic traits are not significantly different from controls in the majority of these relatives. The current study investigated the heterogeneity of autistic traits using latent class analysis (LCA) of the Autism Spectrum Quotient (AQ) ratings of 673 parents of children with ASD and 147 parents of typically developing children. Two distinct subgroups, including a "lowscoring" and a "high-scorer (BAP)" groups, were found. In comparison to control parents, a significantly larger proportion (21.1\% vs $7.5 \%)$ of parents of ASD were members of BAP group. Communication subscale made a distinctive contribution to the separation of high and low-scoring groups $(\mathrm{d}=2.77)$. Further studies investigating neurobiological and genetic biomarkers and stability of these two subgroups over time are important for understanding the nature of autistic traits in the general population.
\end{abstract}

Keywords: Autistic traits; Autism-spectrum questionnaire; latent class analysis; broader autism phenotype 


\section{Introduction}

One of the central features of autism spectrum disorder (ASD) is the significant variability in symptom severity in clinical populations. ASD is a neurodevelopmental disorder characterized by deficits in socialization, communication, and by repetitive behaviours and restricted and circumscribed interests (APA, 2013). ASD has a significant heritability (Freitag et al 2010; Tick et al. 2015). The risk of ASD is significantly increased in first-degree relatives of affected probands (Bolton et al 1994; Constantino et al. 2010; Colvert et al 2015). However, most common phenotypic presentation of genetic susceptibility to ASD is subclinical autistic traits rather than the full-blown clinical disorder. These subclinical traits have been termed as the lesser variant in the earlier studies and as the extended or broader autism phenotype (BAP) in the more recent work (Losh et al 2008; Szatmari et al 2000). Initially, BAP was assessed by family history interview (Bolton et al 1994; Piven et al 97; Szatmari et al 2000) and other time-intensive interview methods including the Pragmatic Rating Scale (Landa et al 1992) and the Broad Autism Phenotype Symptom Scale (Sung et al 2005). In the more recent studies, a number of reliable and more easily administered instruments including the Social Responsiveness Scale, the Autism Spectrum Quotient (AQ), the Broad Autism Phenotype Questionnaire (BAPQ) were used (Baron-Cohen et al 2001; Constantino et al 2010; Hurley et al 2007).

A number of family studies have shown that first-degree relatives of probands with ASD had higher ratings than healthy controls in instruments assessing BAP ( Bishop et al 2004; Wheelright et al 2010, Kose et al 2013; Kadak et al 2014). However, it is important to note that effect sizes of such impairments are rather small and most first-degree 
relatives of probands with ASD have no features of BAP. These findings suggest that there is a significant heterogeneity of subclinical autistic traits among family members of individuals with ASD. Investigating potential subgroups of individuals with distinct profiles of scores in instruments assessing subclinical autistic traits within relatives of ASD can help to define the boundaries of BAP.

Several studies have investigated potential subgroups based on subclinical autistic traits among adult relatives of probands with ASD with the aim of defining boundaries and prevalence of BAP. Several of these studies have categorised parents of ASD into BAP and unaffected groups based on arbitrary cut-off scores of BAP measures. Sason et al (2013) used 1.5 SD above the mean BAPQ score of comparison sample as the threshold of having BAP. This study reported that between $14-23 \%$ for parents of ASD and between $5-9 \%$ for control parents had BAP. Several other studies have also used 1.5 SD above the mean of comparison subjects as the cut-off value of BAP (Losh et al 2008: Piven and Palmer, 1999). Wheelwright et al (2010) had arbitrarily divided BAP into 3 different levels based on the severity of the AQ scores including BAP (1 to 2 SDs above the mean on the AQ), MAP (medium autism phenotype; individuals scoring between 2 to 3 SDs above the mean ) and NAP (narrow autism phenotype; individuals scoring $\geq 3$ SDs above the mean). In this study, ASD fathers had the highest percentage of high-scorers across the BAP, MAP and NAP range (33\%), compared with $23 \%$ of ASD mothers, $22 \%$ of control fathers and $9 \%$ of control mothers (Wheelwright et al 2010). Ruta et al 2012 used a very similar approach with AQ in a Sicilian population and found that $13.5 \%$ of ASD parents and $1 \%$ control parents had MAP or NAP. However, 
there was no significant difference in the proportion of individuals scoring 1 to 2 SDs above the mean of the AQ between the parent groups.

Data-driven methods, including cluster analysis and methodologically more robust latent class analysis (LCA), can provide a more objective classification of subgroups than arbitrary cut-off scores in heterogeneous populations. There is a paucity of studies attempting to identify BAP subgroup among relatives with a data-driven approach. One particular exception is the recent study of Ruzich et al (2015) that used hierarchical cluster analysis in siblings of individuals with ASD and found 2 clusters including low and high scorers of AQ. In Ruzich et al 2015, 27. $4 \%$ males and $16.8 \%$ females were members of high scorers cluster based on the parent-rated AQ scores.

In the current study, our aim was to investigate potential subgroups of parents based on subclinical autistic traits as measured by the AQ using LCA. We hypothesized that these subgroups would include a cluster or clusters of high scorers which will be significantly over-represented among parents of ASD compared to parents of typically developing children.

\section{Methods}

673 parents (359 mothers, 314 fathers) of 360 children with ASD and 146 parents (77 mothers, 69 fathers) of 103 typically developing children included in the study. Parents of children with ASD were recruited from education centres for children with autism or learning and rehabilitation centres in Istanbul or from the pool of two outpatient services of Child and Adolescent Psychiatry departments (in Ege University Medical School or Istanbul University Cerrahpaşa Medical School). All of the children with ASD in this 
study were initially diagnosed by their referring team including a consultant child psychiatrist. All of the children with ASD in this study were also assessed by Disability Boards of the University and State hospitals (including a child psychiatrist, child neurologist and seven other members) for state-funded access to learning and rehabilitation centres in Istanbul and Izmir and were referred to these centers with diagnosịs of ASD based on ICD-10. The study sample mainly included children with a significant need for rehabilitation and diagnosis of childhood autism (F84.0) $(\mathrm{n}=346)$. Six of the children had the diagnosis of Asperger syndrome (F84.5) and 8 children were diagnosed with atypical autism (F84.1). Additionally, the severity of autistic symptoms in children diagnosed with ASD was assessed by Turkish version of Gilliam Autism Rating Scale-Second Edition (GARS-2) which is a structured parent interview tool (Gilliam, 2006; Diken et al 2012). The mean score of Autism Index of GARS-2 was 98.5 $(\mathrm{SD}=15.4)$ which was very similar to mean of GARS-2 (mean=100, $\mathrm{SD}=15)$ in the normative sample for children with autism (Gilliam, 2006). $16.6 \%$ of children diagnosed with ASD had relatively less severe symptoms in GARS-2 (Autism index less than 85) compared to other children with ASD. There was no significant group difference for age between children ASD and typically developing children (Table S1 in supplement). The parents of typically developing children were recruited from the hospital staff and their friends/relatives. Having a history of psychiatric and neurological illness, having a score higher than 29 (mean score $=21.9, \mathrm{SD}=3.8)$ in Childhood Autism Rating Scale (CARS) (Schopler et al 2007) and reporting behavioral problems in Child-Behaviour Check List were exclusion criteria for typically developing children. There was no significant differences in age and gender between parents of ASD children and control 
parents (Table-1). Written informed consent was sought from all participants and procedures followed in the study were approved by local research ethics committees. All included parents completed the Turkish version of AQ (AQ-TR). The AQ is a reliable, standardized, brief and easy to use a self-reported questionnaire (Baron-Cohen et al 2001) for assessing the broader phenotype in adults. It consists of 50 questions, including assessing five different areas: social skill; communication skills; imagination abilities; attention switching; and attention to detail. Each of the items listed here scores 1 point if the respondent records the abnormal or autistic-like behavior either mildly or strongly. The total score of the AQ ranges from 0 to 50 . The maximum score for each of the subscales is 10 . In the original study average score in the control group was 16.4 and individual who scored 32 or more had a high probability of having a clinical ASD diagnosis (Baron-cohen et al 2001). The AQ was translated into Turkish (AQ-TR) by Köse et al (2010).

\section{Data analysis}

Statistical analyses were performed using SPSS (Statistical Package for the Social Sciences) version 11 and R. Normality of distribution of the AQ total score and five subscales were assessed with stem-and-leaf plots and skewness and kurtosis of the data. Skewness (ranged from 0.01 to 0.61 ) and Kurtosis (ranged from -0.44 to 0.26 ) values and inspection of stem and leaf plots suggested that AQ total score and subscale scores had normal distributions. Group differences for demographic variables and AQ scores were tested with t-tests, chi-square tests and two-way ANOVA (significance level of $\mathrm{p}<0.05$ ). Effect sizes of AQ scores were calculated based on Cohen d (mean difference divided by 
pooled standard deviation) formula. We also calculated Z-scores of the AQ measures for both clusters (mean difference divided by standard deviation of control parents).

We performed latent class analysis (LCA) to investigate the clustering of data with Mclust package in R environment (Fraley et al. 2012) in the full study sample ( $\mathrm{n}=819)$.

Mclust is a package for model-based clustering, classification and density estimation and is based on finite Gaussian mixture modelling fitted via EM algorithm. In Mclust, optimal model and number of clusters are selected according to Bayesian information criterion (BIC). As a model-based approach, LCA has a number of advantages over (r) traditional clustering methods such K-means clustering including LCA yielding a smaller misclassification rate and its ability to choose the number of clusters more objectively based on the tests of goodness of fit (Fraley and Raftery, 2002; Magidson and Vermunt, 2002).

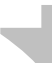

\section{Results}

Full group analysis

Parents of children with ASD had significantly higher scores than control parents on the AQ total and 3 subscales (social skills, communication and imagination) (Table-1) and effect sizes for between-group differences for these measures were modest $(d=0.36-0.56)$. Gender has a significant effect (males scoring higher) on communication subscale only $(\mathrm{F}=5.8, \mathrm{p}=0.02)$, however, there was no significant gender-group interaction effect for communication subscale $(\mathrm{F}=1.4, \mathrm{p}=0.24)$.

Latent class analysis 
In LCA, based on BIC values for multiple models and shape, two-clusters provided the best fit (Figure-1). One cluster (Low-scoring group) included 665 individuals with average scores in AQ. The second cluster (High-scoring group (BAP)) included 154 individuals with high AQ scores. Individuals in BAP cluster had significantly higher scores in the AQ total and all subscales but attention to detail (Table-2; Figure-2) compared to the low-scoring group. The effect-sizes for between-group differences for the communication subscale was very large $(d=2.77)$ and was large for the AQ total $(\mathrm{d}=1.21)$. Effect sizes for between-group differences for social skills, imagination and attention switching were small to medium $(\mathrm{d}=0.26-0.72)$. There were no between-group significant differences for age but the percentage of males were modestly but significantly higher in BAP than low-scoring group (55.5\% vs $44.7 \%, \mathrm{p}=0.01)$. Autistic symptoms severity ratings of children with ASD had no significant effect on cluster membership of their parents. The proportion of parents who were the members of BAP cluster were similar in parents of ASD children with less and more severe autism ratings $\left(20.0 \%\right.$ vs $\left.21.6 \%, \chi^{2}=0.1, p=0.72\right)$. In comparison to control parents, significantly larger proportion ( $21.1 \%$ vs $7.5 \%$ ) of parents of children with ASD were members of BAP group $\left(\chi^{2}=14.8, p<0.001\right) .25 .2 \%$ of fathers and $17.8 \%$ of mothers of children with ASD were members of the BAP group. Only $8.1 \%$ of fathers and $5.9 \%$ of mothers in the control group were members of the high-scoring group. Mean AQ and Communication scores of the parents of the children with ASD who were classified as high-scorers were approximately 1.5 standard deviations $(Z=1.49)$ and 2.5 standard deviations $(Z=2.49)$ above control parents (Table-2). 


\section{Discussion}

In the current study, we investigated autistic traits in parents of children with ASD and control parents using LCA of AQ and showed that two distinct subgroups exist. Our findings confirmed our hypothesis. One of these subgroups included high-scorers and the other cluster included low-scorers and a significantly larger proportion of parents of children with ASD compared to control parents were members of the high-scorers group.

In the full-group analysis, as expected, of parents of children with ASD had significantly but modestly (small to medium effect sizes) higher scores on the AQ and social skills, communication and imagination subscales. These results are supportive of findings of previous studies (Bishop et al 2004; Köse et al 2013). However, more originally, we examined the subgroups of autistic traits with a data-driven method and were able to identify a BAP cluster. The percentage of parents of children with ASD allocated to the BAP cluster were consistent with findings of previous studies using arbitrary cut-off scores (Sasson et al 2013; Ruta et al 2012; Wheelright et al 2010). It seems that phenotypic characteristics of BAP can be detectable in approximately one-fifth of firstdegree relatives of individuals with ASD in adults, as well as in childhood (Georgides et al 2013). Our findings were also generally similar to finding of Ruzich et al (2015) which explored autistic traits subgroups with hierarchical clustering in siblings of individuals with ASD. However, there was one important difference. Ruzich et al (2015) reported that scores in AQ subscales in the high-scoring group was globally high and no subscale of AQ was standing out as predictors of group differences between clusters. In 
contrast, our finding suggested that communication subscale of AQ was distinctively contributing to group differences between high and low-scoring subgroups. Impaired language is one of the defining features of autism and pragmatic language deficits leading to ineffective communication with others and difficulties in understanding indirect speech are important elements of BAP (Landa et al 92 ; Gokcen et al 2009). Family studies examining parents and other relatives of individuals with ASD have reported increased rates of delays in language development (August et al., 1981; Steffenburg et al., 1989; Szatmari et al., 2000). Communication difficulties assessed by interviews and self-report measure have consistently showed difficulties in social (pragmatic) aspects of language in parents and siblings of individuals with ASD (Landa et al 1992; Bolton et al 1994, Ruser et al 2007, Stone et al 2007, Piven et al 1997; Di Michele et al 2007, Whitehause et al 2007). Our findings suggest that communication deficits might be the most sensitive aspect of self-rated AQ to identify BAP. It is also important to consider that social (pragmatic) communication disorder (Swineford et al 2014) can be more common among first-degree relatives of individuals with ASD. Genetic susceptibility to both ASD and social communication disorders can be expressed as pragmatic language difficulties. Importantly, videotapes of conversational interviews showed that $15 \%$ parents of children with specific language impairments and parents of children with ASD have severe deficits in communication (Ruser et al 2007). It might be likely that some of the parents in Class II can meet the diagnostic criteria of social communication disorder.

Gender had a significant but modest effect on the current findings. Communication subscale was the only measure that males reported more difficulties than females. 
However, there was no gender $\mathrm{x}$ group interaction suggesting that gender-related differences were equally relevant for both parent groups. As communication subscale had a distinct effect on separation of low and high-scoring groups, a relatively higher percentage of fathers were members of high-scoring (BAP) group. This finding is consistent with results of previous studies (Ruzich et al 2015). However, a substantial minority of not only fathers but also mothers of children with ASD (17.8 \%) were (n) members of the high-scoring group. Our findings also suggested that attention to detail was not sensitive measure to detect BAP among parents of children with ASD.

The current study has several limitations. Most importantly, this was a cross-sectional study. There is a need for further studies investigating the stability of BAP cluster over time. Self-report nature of assessment is another limitation. Some parents in the highscoring group can potentially meet diagnostic criteria for ASD and future work should consider a consider assessing parents further using standardized measures such as the ADOS/ADI$\mathrm{R}$. As a future direction, it is important to investigate genetic and neurobiological markers of membership to BAP cluster among first-degree relatives of individuals with ASD. 


\section{References}

American Psychiatric Association. (2013). Diagnostic and statistical manual of mental disorders (5th ed.). Arlington, VA: American Psychiatric Publishing.

August, G., Stewart, M., Tsai, L. (1981). The incidence of cognitive disabilities in the siblings of autistic children. British Journal of Psychiatry, 138, 416-422.

Baron-Cohen, S., Wheelwright, S., Skinner, R., Martin, J., Clubley, E. (2001) The autism-spectrum quotient (AQ): Evidence from Asperger syndrome/high-functioning autism, males and females, scientists and mathematicians. Journal of Autism and Developmental Disorders, 31, 5-17.

Bishop, D.V., Maybery, M., Maley, A., Wong, D., Hill, W., Hallmayer, J. (2004). Using self-report to identify the broad phenotype in parents of children with autistic spectrum disorders: a study using the Autism-Spectrum Quotient. Journal of Child Psychology and Psychiatry, 45, 1431-6.

Bolton, P., Macdonald, H., Pickles, A., Rios, P., Goode, S., Crowson, M., et al. (1994). case-control family history study of autism. Journal of Child Psychology and Psychiatry, $35,877-900$.

Colvert, E., Tick, B., McEwen, F., Stewart, C., Curran, S.R., Woodhouse, E., et al (2015). Heritability of Autism Spectrum Disorder in a UK Population-Based Twin Sample. JAMA Psychiatry, 72, 415-23.

Constantino, J.N., Przybeck, T., Friesen, D., Todd, R.D. ( 2000). Reciprocal social behaviour in children with and without pervasive developmental disorders. Journal of Developmental and. Behavioral Pediatritcs, 21, 2-11.

Constantino, J.N., Zhang, Y., Frazier, T., Abbacchi, A.M., Law, P (2010). Sibling recurrence and the genetic epidemiology of autism. American Journal of Psychiatry, 167, 1349-1356.

Di Michele, V., Mazza, M., Cerbo, R., Roncone, R., Casacchia, M. (2007). Deficits in pragmatic conversation as manifestation of genetic liability in autism. Clinical Neuropsychiatry: Journal of Treatment Evaluation, 4 , 144-151. 
Diken, I.H., Ardic, A., Diken, Ö., Gilliam, J.E. (2012). Exploring the Validity and Reliability of Turkish Version of Gilliam Autism Rating Scale-2: Turkish Standardization Study. Education \& Science, 37, 318-328.

Fraley, C., Raftery, A.E. (2002). Model-based Clustering, Discriminant Analysis and Density Estimation. Journal of American Statistics Association, 97, 611-631

Fraley, C., Raftery, A.E., Murphy, T.B., Scrucca, L. ( 2012). Mclust Version 4 for R: Normal Mixture Modeling for Model-Based Clustering, Classification, and Density Estimation. Technical Report No. 597, Department of Statistics, University of Washington

Freitag, C.M., Staal, W., Klauck, S.M., Duketis, E., Waltes, R (2010). Genetics of autistic disorders: review and clinical implications. European Child and Adolescence Psychiatry, $19,169-78$.

Georgiades, S., Szatmari, P., Zwaigenbaum, L., Bryson, S., Brian, J., Roberts, W., et al (2013). A prospective study of autistic-like traits in unaffected siblings of probands with autism spectrum disorder. JAMA Psychiatry, 70, 42-8.

Gilliam, J.E., (2006). Gilliam Autism Rating Scale: Second Edition. Austin, TX.: PROED.

Gokcen, S., Bora, E., Erermis, S., Kesikci, H., Aydin, C. (2009). Theory of mind and verbal working memory deficits in parents of autistic children. Psychiatry Research, 166, 46-53.

Hurley, R.S., Losh, M., Parlier, M., Reznick, J.S., Piven, J. (2007). The broad autism phenotype questionnaire. Journal of Autism and Developmental Disorders, 37, 16791690.

Kadak, M.T., Demirel, O.F., Yavuz, M., Demir, T. (2014). Recognition of emotional facial expressions and broad autism phenotype in parents of children diagnosed with autistic spectrum disorder. Comprehensive Psychiatry, 2014, 55, 1146-51.

Köse, S., Bora, E., Erermis, S., Aydın, C. (2010). Psychometric features of Turkish version of autism-spectrum quotient. Anadolu Psikiyatri Dergisi, 11, 253-260 (in Turkish).

Köse, S., Bora, E., Erermiş, S., Özbaran, B., Bildik, T., Aydın, C. (2013). Broader autistic 
phenotype in parents of children with autism: Autism Spectrum Quotient-Turkish version. Psychiatry and Clinical Neuroscience, 67(1), 20-7.

Landa, R., Piven, J., Wzorek MM, Gayle JO, Chase GA, Folstein SE. (1992). Social language use in parents of autistic individuals. Psychological Medicine, 1992 ;22:245-54. Losh, M., Childress, D., Lam, K., Piven, J. (2008). Defining key features of the broad autism phenotype: a comparison across parents of multiple- and single-incidence autism families. American Journal of Medical Genetics Part B Neuropsychiatric Genetics, 147B, $424-433$.

Magidson, J., Vermunt, J. (2002) Latent class models for clustering: a comparison with K-means. Can J Market Res, 20, 36-43

Piven, J., Palmer, P., Landa, R., Santangelo, S., Jacobi, D., Childress, D. (1997).

Personality and language characteristics in parents from multiple-incidence autism families. American Journal of Medical Genetics, 74, 398-411.

Piven, J., Palmer, P. (156). Psychiatric disorder and the broad autism phenotype:

evidence from a family study of multiple-incidence autism families. American Journal of Psychiatry, 156(4), 557-63.

Ruser, T.F., Arin, D., Dowd, M., Putnam, S., Winklosky, B., Rosen-Sheidley, B., et al (2007). Communicative competence in parents of children with autism and parents of children with specific language impairment. Journal of Autism and Developmental Disorders, 37, 1323-36.

Ruta, L., Mazzone, D., Mazzone, L., Wheelwright, S., Baron-Cohen, S. (2012). The Autism-Spectrum Quotient--Italian version: a cross-cultural confirmation of the broader autism phenotype. Journal of Autism and Developmental Disorders, 2012, 42:625-33. Ruzich, E., Allison, C., Smith, P., Watson, P., Auyeung, B., Ring, H., et al (2015). Subgrouping siblings of people with autism: Identifying the broader autism phenotype. Autism Research, doi: 10.1002/aur.1544

Sasson, N.J., Lam, K.S., Childress, D., Parlier, M., Daniels, J.L., Piven, J. (2013). The broad autism phenotype questionnaire: prevalence and diagnostic classification. Autism Research, 6, 134-43.

Schopler, E., Reichler, R.J., Rochen Renner, B. (2007). The Childhood Autism Rating Scale (CARS), eleventh printing, Western Psychological Services. 
Steffenburg, S., Gillberg, C., Hellgren, L., Andersson, L., Gillberg, I.C., Jakobsson, G., et al (1989). A twin study of autism in Denmark, Finland, Iceland, Norway and Sweden. Journal of Child Psychology and Psychiatry 30, 405-416.

Stone, W.L., McMahon, C.R., Yoder, P.J., Walden, T.A. (2007). Early socialcommunicative and cognitive development of younger siblings of children with autism spectrum disorders. Archives of Pediatric and Adolescence Medicine, 161, 384-90.

Swineford, L.B., Thurm, A., Baird, G., Wetherby, A.M., Swedo, S. (2014). Social (pragmatic) communication disorder: a research review of this new DSM-5 diagnostic category. Journal of Neurodevelopmental Disorders, 6, 41.

Szatmari, P., MacLean, J.E., Jones, M.B., Bryson, S.E., Zwaigenbaum, L., Bartolucci, G, et al. (2000). The familial aggregation of the lesser variant in biological and nonbiological relatives of PDD probands: A family history study. Journal of Child Psychology and Psychiatry 41, 579-586.

Sung, J.U., Dawson, G., Munson, J., Estes, A., Schellenberg, J., Wijsman, E.M. (2005).

Genetic investigation of quantitative traits related to autism: Use of multivariate polygenic models with ascertainment adjustment. American Journal of Human Genetics $76,68-81$.

Tick, B., Bolton, P., Happé, F., Rutter, M., Rijsdijk, F (2015). Heritability of autism spectrum disorders: a meta-analysis of twin studies. Journal of Child Psychology and Psychiatry, doi: 10.1111/jcpp.12499

Wheelwright, S., Auyeung, B., Allison, C., Baron-Cohen, S. (2010). Defining the broader, medium and narrow autism phenotype among parents using the Autism Spectrum Quotient (AQ). Molecular Autism 1, 10.

Whitehouse, A.J., Barry, J.G., Bishop, D.V. (2007).The broader language phenotype of autism: a comparison with specific language impairment. Journal of Child Psychology and Psychiatry 48, 822-30. 


\section{Figure Legends}

Figure 1 BIC values of latent class analyses for different solutions (1 to 9 classes)

Figure 2 Means of AQ subscales in class 1 and class 2
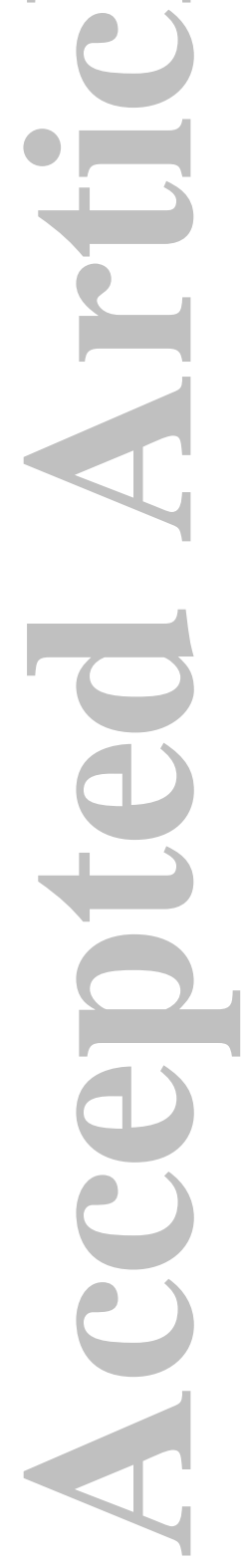

John Wiley \& Sons

This article is protected by copyright. All rights reserved. 


\section{Table Legends}

Table 1-Characteristics of parents of ASD and TD children

Table 2-Characteristics of parents in cluster 1 and 2

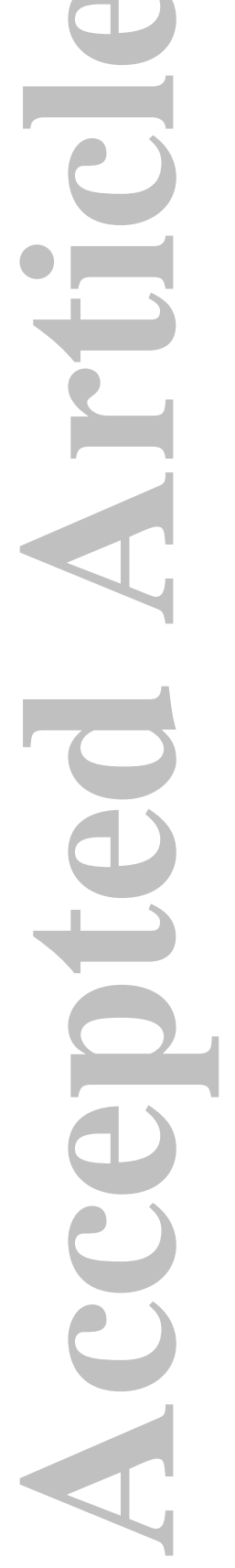

John Wiley \& Sons

This article is protected by copyright. All rights reserved. 


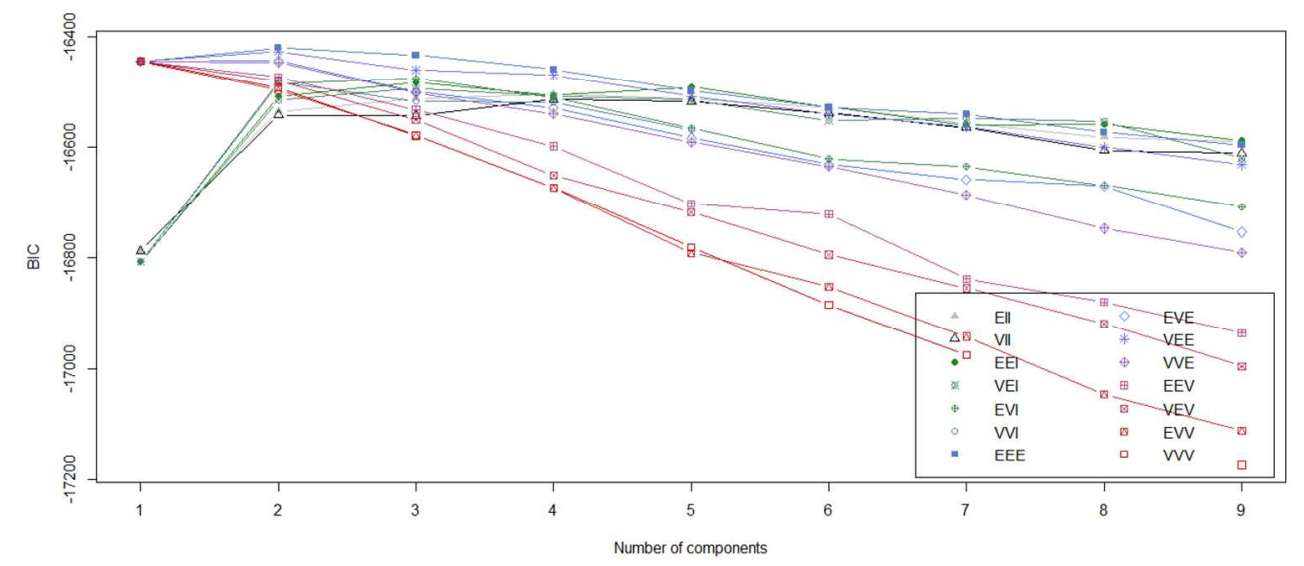

Figure 1

$481 \times 229 \mathrm{~mm}(72 \times 72 \mathrm{DPI})$

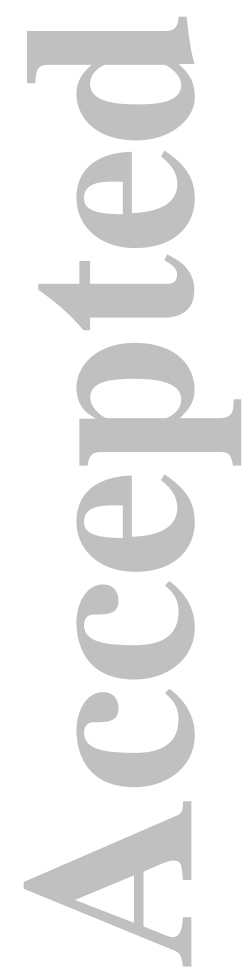

John Wiley \& Sons

This article is protected by copyright. All rights reserved. 


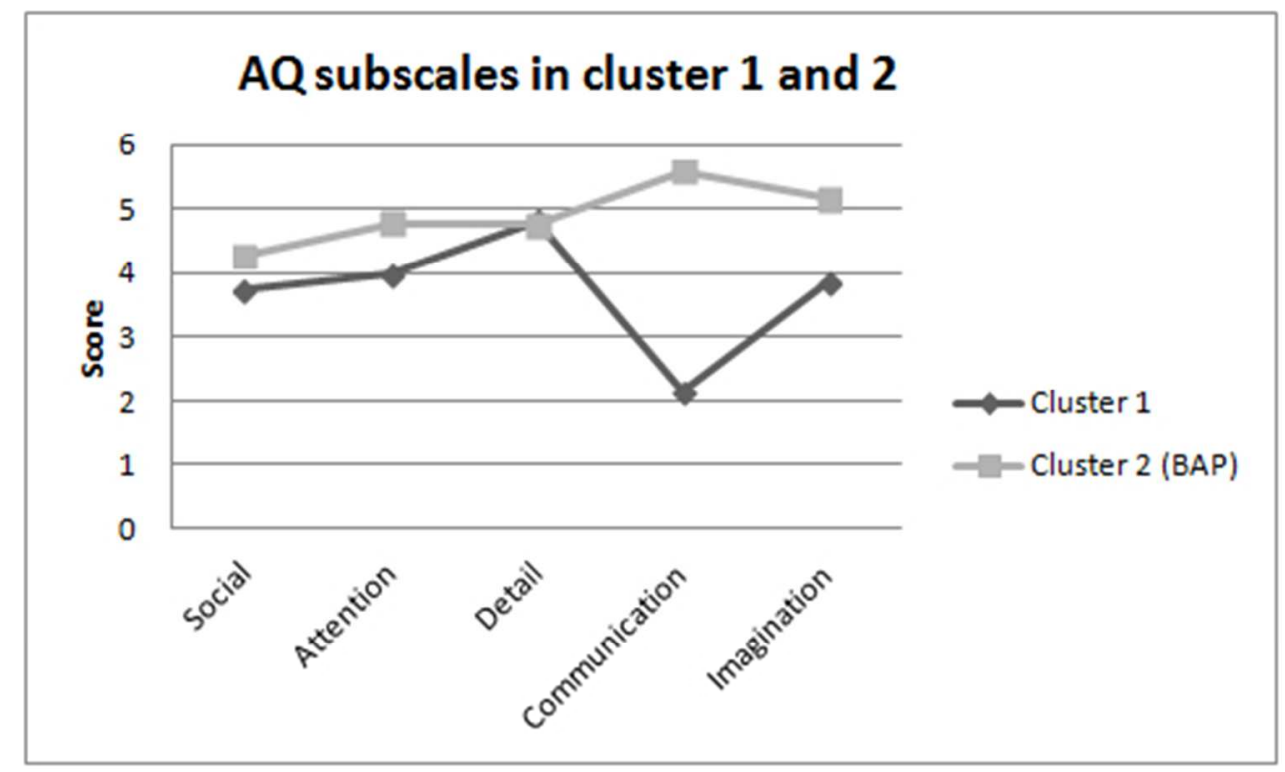

Figure 2

$170 \times 102 \mathrm{~mm}(72 \times 72 \mathrm{DPI})$

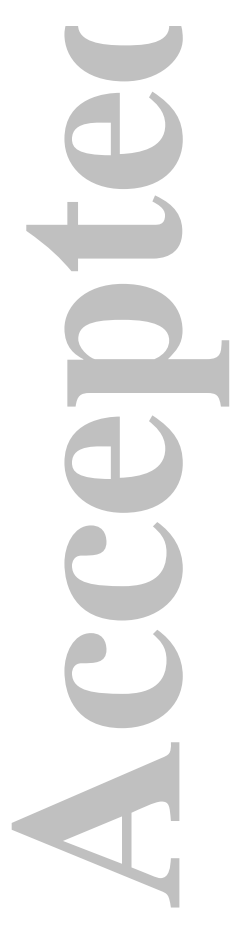

John Wiley \& Sons

This article is protected by copyright. All rights reserved. 
Table 1-Characteristics of parents of ASD and TD children

\begin{tabular}{|l|l|l|l|l|l|}
\hline & ASD & TD & Statistics & $\mathrm{p}$ & Cohen D \\
\hline N (male \%) & $673(46.7 \%)$ & $146(47.3 \%)$ & $\chi^{2}=0.02$ & 0.90 & \\
\hline Age & & & $\chi^{2}=0.02$ & 0.14 & \\
\hline 25 or younger & $2.5 \%$ & $3.5 \%$ & & & \\
\hline $26-30$ & $9.7 \%$ & $16.0 \%$ & & & \\
\hline $32-35$ & $23.8 \%$ & $26.4 \%$ & & & \\
\hline $36-40$ & $29.8 \%$ & $28.5 \%$ & & & \\
\hline 41 or older & $34.1 \%$ & $25.7 \%$ & & & \\
\hline & Mean (SD) & Mean (SD) & & & \\
\hline AQ total & $20.1(5.5)$ & $17.4(5.0)$ & $\mathrm{F}=33.2$ & $<0.001$ & 0.50 \\
\hline Social & $4.04(1.92)$ & $2.98(1.76)$ & $\mathrm{F}=38.1$ & $<0.001$ & 0.56 \\
\hline Attention & $4.18(1.76)$ & $3.99(1.88)$ & $\mathrm{F}=1.74$ & 0.19 & 0.11 \\
\hline Detail & $4.81(1.93)$ & $4.83(1.87)$ & $\mathrm{F}=0.02$ & 0.89 & -0.01 \\
\hline Communication & $2.95(1.87)$ & $2.05(1.44)$ & $\mathrm{F}=27.3$ & $<0.001$ & 0.50 \\
\hline Imagination & $4.23(1.88)$ & $3.55(1.83)$ & $\mathrm{F}=16.1$ & $<0.001$ & 0.36 \\
\hline
\end{tabular}

$\mathrm{TD}=$ Typically developing, $\mathrm{ASD}=$ Autism-spectrum disorder
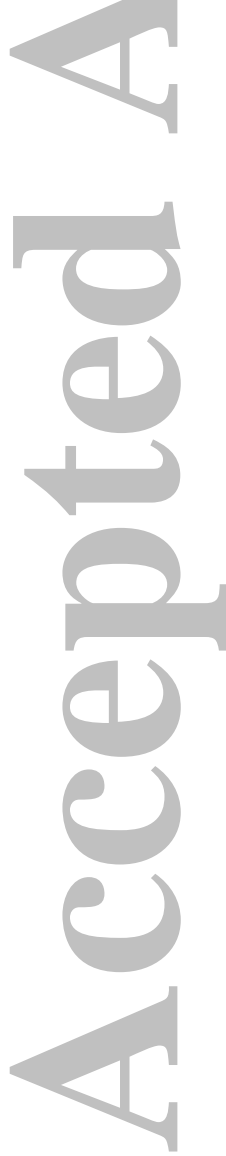
Table 2-Characteristics of parents in cluster 1 and 2

\begin{tabular}{|c|c|c|c|c|c|c|c|}
\hline & Class 1 & Class 2 (BAP) & Statistics & $\mathrm{p}$ & $\begin{array}{l}\text { Class } \\
2 \text { vs } 1\end{array}$ & Class $2^{\mathrm{ASD}}$ & $\begin{array}{l}\text { Class } 2^{\text {ASD }} \\
\text { vs controls }\end{array}$ \\
\hline $\mathrm{N}($ male $\%)$ & $665(44.7 \%)$ & $154(55.8 \%)$ & $\chi^{2}=6.28$ & 0.01 & & $143(55.2 \%)$ & \\
\hline Age & & & $\chi^{2}=2.43$ & 0.79 & & & \\
\hline 25 or younger & $2.7 \%$ & $2.6 \%$ & & & & & \\
\hline $26-30$ & $10.8 \%$ & $11.2 \%$ & & & & & \\
\hline $32-35$ & $23.8 \%$ & $26.3 \%$ & & & & & \\
\hline $36-40$ & $30.6 \%$ & $25.0 \%$ & & & & & \\
\hline 41 or older & $32.1 \%$ & $34.9 \%$ & & & & & \\
\hline & Mean (SD) & Mean (SD) & & & Cohen D & Mean (SD) & $\mathrm{Z}($ Cohen $\mathrm{D})$ \\
\hline AQ total & $18.58(4.99)$ & $24.59(4.85)$ & $t=13.5$ & $<0.001$ & 1.21 & $24.84(4.88)$ & $1.49(1.51)$ \\
\hline Social & $3.76(1.92)$ & $4.27(1.96)$ & $\mathrm{t}=3.0$ & 0.003 & 0.26 & $4.38(1.92)$ & $0.80(0.76)$ \\
\hline Attention & $3.99(1.78)$ & $4.78(1.66)$ & $\mathrm{t}=5.0$ & $<0.001$ & 0.45 & $4.77(1.69)$ & $0.41(0.44)$ \\
\hline Detail & $4.82(1.95)$ & $4.77(1.77)$ & $\mathrm{t}=0.32$ & 0.75 & -0.03 & $4.76(1.81)$ & $-0.04(-0.04)$ \\
\hline Communication & $2.15(1.26)$ & $5.59(1.16)$ & $\mathrm{t}=31.0$ & $<0.001$ & 2.77 & $5.64(1.18)$ & $2.49(2.72)$ \\
\hline Imagination & $3.87(1.80)$ & $5.18(1.89)$ & $\mathrm{t}=8.0$ & $<0.001$ & 0.72 & $5.27(1.89)$ & $0.89(0.88)$ \\
\hline
\end{tabular}

Class $2^{\mathrm{ASD}}=$ Only parents of children with ASD in class 2 ; BAP=Broader autism phenotype

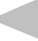

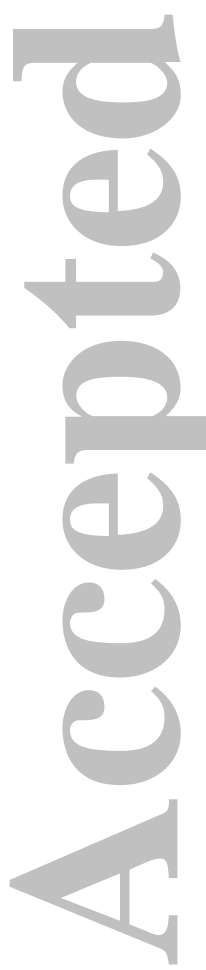




\section{University Library}

\section{- M I I N E R VA \\ A gateway to Melbourne's research publications}

Minerva Access is the Institutional Repository of The University of Melbourne

Author/s:

Bora, E;Aydin, A;Sarac, T;Kadak, MT;Kose, S

Title:

Heterogeneity of subclinical autistic traits among parents of children with autism spectrum disorder: Identifying the broader autism phenotype with a data-driven method

Date:

2017-02-01

\section{Citation:}

Bora, E., Aydin, A., Sarac, T., Kadak, M. T. \& Kose, S. (2017). Heterogeneity of subclinical autistic traits among parents of children with autism spectrum disorder: Identifying the broader autism phenotype with a data-driven method. AUTISM RESEARCH, 10 (2), pp.321-326. https://doi.org/10.1002/aur.1661.

Persistent Link:

http://hdl.handle.net/11343/291935 